\title{
Regional Dysregulation and Aberrant Functional Connectivity in ADHD
}

\author{
Archer $\mathrm{T}^{1^{*}}$ and Abedini $\mathrm{Y}^{2}$ \\ ${ }^{1}$ Department of Psychology, University of Gothenburg, Sweden \\ ${ }^{2}$ Specialist Centre, Child and Juvenile Angereds Hospital, Gothenburg, Sweden
}

*Corresponding author: Archer T, Department of Psychology, University of Gothenburg, Sweden, E-mail: trevor. archer@psy.gu.se

Citation: Archer T and Abedini Y (2016) Regional Dysregulation and Aberrant Functional Connectivity in ADHD. J Neurosci Neuropsyc 1: 101. doi: 10.18875/2577-7890.1.101

Article history: Received: 25 November 2016, Accepted: 30 January 2017, Published: 02 February 2017

\begin{abstract}
The trajectory of neurodevelopmental adversity determining attention-deficit/hyperactivity disorder (ADHD) from childhood to adulthood which is recognized increasingly by the long-term impairment and the burgeoning persistence beyond childhood, through adolescence, to adulthood poses a prolonged history defined by a concatenation of symptom profiles and an underlying dysregulation accompanied by aberrant brain regional networks. Abnormalities within the "default mode network" and disharmonizing fluctuations afflicting brain regional connectivity, even inducing dysconnectivity, appear to be associated both with symptoms profiles and the relative efficacy of putative interventional outcomes. Co-morbidity in ADHD, a persistently debilitating and potentially regressive issue, is viewed from a perspective of eating disorders and substance use disorders although several other combinations proliferate. Among the factors contributing to dysregulation and aberrant connectivity in brain development are included genetic and epigenetic proclivities, neurotoxins and neurodegeneration, predisposing characteristics and environmental pressures. Finally, the likelihood of perinatal stress trauma and/or inflammation as contributory circumstances ought to be considered.
\end{abstract}

Keywords: ADHD; Childhood; Adolescence; Adulthood; Dysregulation; Aberrant connectivity; Default mode; Biomarkers; Comorbidity; Perinatal

Attention-deficit/hyperactivity disorder (ADHD) prevalence appears, by most current accounts, to be steadily increasing, possibly due to greater diagnostic, parental and teacher awareness but also to the interactive effects of environmental stressors, chemical agents and a generally disharmonious and unhealthy lifestyles that major portions of the populations have adopted (Archer et al., 2011).[1] In many respects, the burgeoning incidence of ADHD and accompanying health problems is alarming (Archer and Garcia, 2016),[2] despite the in-roads that have been made pertaining to intervention (Archer and Kostrzewa, 2012)[3]. ADHD symptom profiles vary between children, adolescents and adults but basically incorporate an exaggerated level of impulsiveness, lack of attention and a high degree of distractibility, excessive thoughtlessness and forgetfulness, losing things and disability in 'starting-and-finishing' (Archer and Garcia, 2016)[2], as well as alterations of the processes determining decision-making and reinforcement learning. ADHD pathophysiology incorporates a concatenation of multiple, cohesive modality spanning deficits, with each deficit expressing marked spatial overlap within patterns of structural and functional dysregulation (Kessler et al., 2014; Metin et al., 2015)[4,5]. A growing consensus indicates that as 'part-and-parcel' to the ADHD condition impairments, the default network-ventral attention network interconnections, or dysconnections, present a key locus of dysfunction and neurodevelopmental derangement (Sripada et al., 2014a,b).[6] Kessler et al. (2016)[7a,ab] have identified parallel associations between intrinsic connectivity networks dysmaturation and ADHD diagnosis in a sample of youths. They observed a pattern depicting a consistent biomarker for severe attentional performance deficits concurrent with a 'down-shifted' expression of intrinsic connectivity network maturation, i.e. shallow maturation, as opposed to a right-shifted expression, i.e. 'lagged' maturation. ADHD children and adolescents demonstrated markedly reduced dorsal caudate functional connectivity with the superior and middle prefrontal cortices as well as reduced dorsal putamen connectivity with the parahippocampal cortex (Hong et al., 2015). [8] Estimations of connectivity correlated in diametrically opposite directions among the ADHD and healthy control individuals, respectively, through the assessment attentional performance by application of the Continuous Performance Test, despite a good response to methylphenidate. Remarkably, it seems the case that studies carried out on ADHD patients to a major extent target upon dopaminergic genes and the structure of basal ganglia using structural and functional Magnetic Resonance Imaging (MRI and fMRI) (Vilor-Tejedor et al., 2016).[9] 
Using fMRI to ascertain expectancy in children presenting ADHD symptoms through assessment of between-group differences in brain activity related to expectations concerning 'when' (i.e. timing), but not 'what' events will occur (i.e. cognitive control). Explicitly, Van Hulst et al. (2016)[10] obtained timing-related hypoactivity which was shown to be partially related to those children presenting a primary diagnosis of ADHD (left hemisphere pallidum) and partially shared by those children presenting similar levels of ADHD symptoms and a primary diagnosis of autism spectrum disorders (left heisphere subthalamic nucleus). Furthermore, they obtained a poorer task performance that was related to timing, but only in the autism spectrum disorder children and symptoms of ADHD. It would seem to be the case that the presence of these neurobiological changes in children presenting ADHD symptoms may be linked to a failure to construct or monitor expectations thereby hindering the efficiency/ efficacy of the child's reciprocal interactions with environmental stimuli. Resting-state fMRI analysis of 56 child and adolescent ADHD patients and 56 normal controls (aged matched) the frontoparietal network which is associated with hyperactivity and impulsiveness expressed marked interactive effects of patient age and symptom profiles predictive for IQ scoring (Park et al., 2016) [11]. Using 3.0 Tesla fMRI scanner, and estimating the Korean ADHD Rating Scale, the Wisconsin Card Sorting Test, the 7- and 14-ring drill test with hop jumps, it found that childhood ADHD patients expressed an extended distance-traveled estimate and a decreased-speed on the 14-ring hop jump task whereas on the Wisconsin Card Sorting Test task, ADHD children demonstrated decreased activation within right gyrus brain region with total distance on the 14-ring hop jump task was negatively correlated with the mean $\beta$ value of Cluster 2 in ADHD children according to the Korean ADHD Rating Scale (Kang et al., 2016)[12]. Despite these indications, using task-based fMRI to assess functional connectivity alterations between key reward processing regions in adolescents and young adults presenting ADHD (Oldehinkel et al., 2016)[13], it appears some manner of 'developmental normalization' occurred or that the reward-processing deficits earlier observed emanated from particular functional connectivity alterations in general task-related networks.

The "default mode network", a regional brain interlocking nexus distinct from other networks, represents a confluence of interacting brain regions, expressing more-or-less synchronically correlated activity, and pathways under activation at times when individuals are unfocused upon the surrounding environment and their brains remain in a state of wakeful rest (Bellana et al., 2016)[14]. It has been found that abnormalities in the intrinsic activity of resting state networks may contribute to the etiology of conduct disorder and poor prognosis of ADHD in combination with conduct disorder (Uytun et al., 2016)[15]; whereas reduced default mode functional connectivity is associated with expressions of conduct disorder (Broulidakis et al., 2016)[16], individuals presenting ADHD present disproportionately high expressions of default mode network activity related to goal-directed tasks accompanied by 'switch-specificity alterations in the right insula region (Sidlauskaite et al., 2016)[17]. Moreover, adult ADHD patients demonstrated markedly stronger resting-state functional connectivity in the anterior cingulate gyrus of the executive control network, as well as in the cerebellar network, than healthy control adults (Moster et al., 2016)[18]. Opposing patterns of functional variability changes have been established for patients presenting schizophrenia spectrum disorders in contrast to those presenting ADHD: the former express lower variability in regions of the default mode network whereas the latter, with autism spectrum disorders, express higher levels of variability; on the other hand, within subcortical regions the former expressed higher variability and the latter lower levels of variability with symptoms scores related to variability levels in each case (Zhang et al., 2016) [19]. In a comparison with age-, gender-, and performance IQ-matched typically developing children, ADHD children showed weaker levels of functional connectivity between the right anterior prefrontal cortex and the right ventrolateral prefrontal cortex, as well as between the left anterior prefrontal cortex and the right inferior parietal lobule (Lin et al., 2015)[20]. In this context, aberrant connectivity represented an etiophysiopathogenic expression of the altered frontoparietal control network in ADHD that are related particular to symptoms of impulsiveness and opposition-defiance (see above); these deficits ought to be observed with reference to those deficits pertaining to response inhibition and attentional control. Nevertheless, this observation renders it somewhat problematic to resolve the greater activated network-wise amplitude of low frequency fluctuations and enhanced functional connectivity variants between the intrinsic connectivity networks together with a temporal pattern within posterior default mode network that correlated positively with inattention scores among ADHD children (Wang and Li, 2015)[21]. Among ADHD adolescents, their unaffected siblings and healthy controls, it was shown that the former two groups presented weaker functional connectivity within the response inhibition network (van Rooij et al., 2015)[22], whereas the latter group showed lesser connectivity between default mode network nodes. Greater response inhibition network connectivity related to lower ADHD severity concurrent with higher levels of default mode connectivity related to elevated ADHD severity.

Brain connectivity assessments cover three basic areas of research: (i) anatomical connectivity, (ii) functional connectivity and (iii) effective connectivity referring to structural aspects of connections between neighboring neurons, the temporal dependency of neuronal activation patterns of anatomically separated brain regions and the particular type of influence that one neuronal system brings to bear upon another type of neuronal system thereby emulating causal interactions between the associated activated brain areas (Lang et al., 2012)[23]. Among the methods applied for studying connectivity may be listed several neuroimaging and neuroanatomical techniques, including: fMRI (see above), diffusion-weighted MRI tractography (Yo et al., 2009)[24], EEG (Zaric et al., 2016)[25], magnetoencephalographic analysis (MEG, Liuzzi et al., 2016)[26], PET (Trotta et al., 2016; Vanicek et al., 2016) [27] and SPECT (Jann et al., 2015)[28]. The quantification of brain connectivity proceeds through the encoding of neighborhood relations into a connectivity matrix by which whose rows and columns correspond to the different brain regions. Functional connectivity is commonly deduced from intervoxel cross-correlations and is often assumed to reflect also the interregional coherence of fluctuations in activity of the underlying neuronal networks within the different brain regions implicated. Currently, 
the separations between anatomical neuronal connections and related functional connectivity and/or effective connectivity remain the focus of much study.

The intimate role of dopaminergic neurotransmission, both due to hereditary and environmental influence, as well as from drugtreatment studies is well-established (Hung et al., 2016; Schrantee et al., 2016 Ziegler et al., 2016)[ 29,30,31] while more recent approaches point to the importance of dopamine-glutamate interactions (Miller et al., 2014)[32]. Several lines of evidence have implied that sporadic disturbances in the signaling of glutamate receptors during a critical period of brain development may contribute to the ADHD pathophysiology (Archer and Garcia, 2016)[2]. It seems likely that the various symptom profiles expressed through behavioral deficits in ADHD are linked to alterations within the global cortico-striatal functional architecture of brain, or whether or not ADHD-related alterations are limited to local, intrastriatal functional connections. Ketamine and other glutamate antagonists have been applied as animal models for ADHD (Fredriksson and Archer, 2002, 2003, 2004; Fredriksson et al., 2004) $[33,34,35,36]$; in this context, it ought to be considered that ketamine caused specific connectivity alterations within and between resting-state consciousness networks with frontoparietal default mode connectivity disruption together with default mode anticorrelation and sensory plus sensory-motor network connectivity preservation unfolding (Bonhomme et al., 2016)[37]. In adult ADHD patients, impaired/altered cerebellar areas of the default mode network have been observed (Hove et al., 2015; Kucyi et al., 2015)[38,39]. A consistent feature of childhood ADHD are the aberrant, dysregulated, cross-network interactions between the salience network, central executive network, and default mode network (Cai et al., 2015)[40]. Serotonergic modulation of functional connectivity appears to be implicated in the resting-state default mode network since acute tryptophan depletion with subsequent losses of serotonin availability to ADHD patients induced reduced functional connectivity in the right superior premotor cortex and left somatosensory cortex, whereas the reverse was observed in the healthy controls (Biskup et al., 2016)[41]. Regarding the serotonin transporter, Vanicek et al. (2016)[42] obtained significant differences in the interregional correlations between the precuneus and the hippocampus in patients with ADHD compared to healthy controls, using the serotonin transporter binding potential, "SERT BPND", of the investigated regions-of-interest under study. Acute methylphenidate administration caused marked functional connectivity alterations between regions of the default mode network with marginal increases in the anterior-posterior connectivity location of the network (Battel et al., 2016)[43].

ADHD predisposes patients to several conditions, e.g. narcolepsy and hypersomnia, but even more important there appears to persist a shared pathophysiology with various addictive syndromes incorporated in the Reward Deficiency Syndrome, such as drug abuse and eating-intake disorders (Modestino et al., 2015)[44]. Furthermore, it has been proposed over a series of reports that genetic variants of dopaminergic genes and other "reward genes" are important common determinants of reward deficiency syndrome (RDS), which we hypothesize includes ADHD as a behavioral subtype (cf. Blum et al., 1996, 2000, 2008a,b, 2010) $[45,46,47,48,49]$. ADHD symptom profiles, particularly regarding the hyperactivity syndromes, attentional problems and impulsiveness, may present surprisingly high levels of co-morbidity to a range of 'parallel' disorders, including eating disorders and drug-related disorders; yet, there has been found to be an uncertain variability in the diagnostic rate of both ADHD and its co-morbidities. For example, a clear positive relationship between responsiveness-to-food and the total ADHD symptom index, especially impulsiveness, lack of attention and the hyperactivity subscales, separately, has been observed (Leventakou et al., 2016)[50]. In a similar context, they obtained also marked positive associations between emotional overeating and ADHD symptoms; those children linked to the highest tertile of the emotional overeating scale presented higher total ADHD index and hyperactivity subscale scores. As an indication of, ADHD children's food avoidant behaviors, 'food fussiness' was shown to be associated significantly with the impulsiveness subscale. The authors found also marked dose-response associations between the expressions of food approach and the ADHD symptoms. Medium level and the highest tertile ADHD children pertaining to the food responsiveness subscale presented elevated scoring on the ADHD total scale, in comparison with those on the lowest tertile. Across gender, individuals presenting lifetime and previous 12-month binge eating and binge eating disorder showed significantly higher prevalence for ADHD diagnosis than individuals without binge eating and binge eating disorder, respectively (Brewerton and Duncan, 2016)[51]. Female ADHD patients presenting lifetime and previous 12-month bulimia nervosa and lifetime anorexia nervosa also showed markedly higher prevalence for ADHD diagnosis in comparison with female patients not presenting these diagnoses. In view of the situation that ADHD expression was invariably initiated earlier than the eating disorder expression, ADHD condition offers an important risk factor for subsequent binge eating and related eating disorder. Co-morbidity with cocaine abuse among adult ADHD diagnosed individuals presented the highest level of effect size for global cognitive impairment compared with cocaine-naïve ADHD patients and healthy controls (Wunderli et al., 2016)[52]. Compared with adult ADHD patients without substance use disorders, patients presenting ADHD together with cocaine/crack showed markedly lower mean IQ levels and higher levels of motor impulsiveness as well as performing more poorly on tasks that assessed verbal skills, vigilance, implicit learning during decision making (Miguel et al., 2016)[53]. The former group of patients (no substance abuse) displayed a worsened level of performance on selective attention, information processing, and visual search tests. Taking converging trends together, Capusan et al. (2016)[54] have collected evidence demonstrating that ADHD symptoms and their associated subtypes derived from the general population were related to increased risks for all types of substance use/abuse disorder outcomes, without any differences between the ADHD subtypes, without substance preferences, and in the absence of gender differences for the presented co-morbidity. Finally, levels of estimated ADHD-symptom severity, co-morbid presence of conduct disorder and major depressive disorder tendency, and treatment-efficacy for ADHD remain the main predictors of ADHD persistence over the childhood-to-adulthood trajectory (Caye et al., 2016)[55]. 
Laboratory animal models of ADHD imply that several factors contribute to the dysregulation and dysconnectivity observed in the expressions of disorder, including:- (i) genetically- and/or epigenetically-based models, e.g. spontaneously hypertensive rats (Kim et al., 2016) [56] or Naples High-excitability rats (Ruocco et al., 2015)[57], SNAP-25-deficient mutant coloboma mice (Baca et al., 2013)[58] or Cadherin-13, risk gene for ADHD (Rivero et al., 2015) [59]; further, Nr3c1-Bhlhb2, transcription factor silencing, axis dysregulation was involved in the development of ADHD through a disruption of prefrontal cortex functioning (Wu et al., 2016)[60]. In a pair-matching case-control study of ADHD children, it was found that Aberrant DNA methylation and histone acetylation was established in ADHD by applying the combination of p300, MYST4 and HDAC1 to obtain an accuracy of 0.9338 (Xu et al., 2015)[61]. (ii) Neurotoxicity- and/or neurodegeneration-based models, e.g. pesticide and perflouroalkyl substances (Lien et al., 2016; Mostafalou and Abdollahi, 2016)[62,63], that implicate both neurons and glial cells (Motaghinejad et al., 2016; Shah and Lahiri, 2016)[64,65]. (iii) N-methyl-D-aspartate (NMDA) neonatally-injected antagonist models in rodents, e.g. NMDA blockade during the brain growth spurt period (Oliveira-Pinto et al., 2015)[66], and single unit recordings of basal and glutamateinduced excitability of pyramidal neurons in the prefrontal cortex of anaesthetised rats coupled with microiontophoresis (Di Miceli and Gronier, 2016)[67], as well as biomorphic electrode concurrent evaluations of glutamate in aligned networks of the frontal cortex that involve the cingulate, prelimbic, infralimbic and dorsal peduncle regions (Miller et al., 2015.[68] (iv) Models based upon changes induced by the environment, e.g. exposure to 'greenhouse gas' (Fluegge, 2016)[69], genetic and environmental overlap during development (Brikell et al., 2016; Homberg et al., 2016)[70,71], childhood trauma history (Ferrer et al., 2016)[72], familial aggregation driven by environmental factors (Chen et al., 2016; Yin et al., 2016)[73,74], peer influence as a magnifier of ADHD diagnosis (Aronson, 2016) [75], second-hand smoking exposure and low blood lead levels (Joo et al., 2016)[76], preschool hyperactivity expression and developmental trajectories (Lopez-Vicente et al., 2016; Smith et al., 2016)[77,78] and susceptibility risk factors (Vilor-Tejedor et al., 2016)[79].

It has been observed that low circulating levels of omega-3 are linked to conditions presenting CNS-related disorders, including cognitive deficit disorders, depression and anxiety disorders, poor anger and conduct control, ADHD and related syndromes, and accelerated neurodegeneration in the elderly individuals (Grant and Guest, 2016) [80]. Similarly, neuroimmune dysregulation occurs among a variety of CNS conditions with interactions involving central and peripheral immune mechanisms that have been found to be disrupted as observed through a series of neuroimmune biomarkers, such as CD3, CD4, CD7, HLA-DR, CD25, CD28, and CD56, which display variability in brain disorders as indicated in anxiety, depression, psychosis, stroke, Alzheimer's disease, Parkinson's disease, attention-deficit hyperactivity disorder, migraine, epilepsy, vascular dementia, mental retardation, cerebrovascular encephalopathy, multiple sclerosis, brain tumors, cranial nerve neuropathies, mental retardation, and posttraumatic brain injury (Cacabelos et al., 2016)[81]. In a functional magnetic resonance imaging study of a cohort of ADHD adolescents compared with matched healthy controls, Von Rhein et al. (2016)[82] did not confirm the notion that ADHD was associated with brain alterations in cortico-striatal networks but rather alterations within local regional striatal functional connectivity thereby implicating aberrant development of local regional functional connectivity of the putamen complex, which could generate eventually the augmentation of functional segregation between the anterior and posterior putamen in the disorder. Higher levels of functional connectivity, particularly within the frontal regions (anterior cingulate cortex) of the brain, linked to the executive control network were observed to correspond to reductions in ADHD symptoms with regard to hyperactivity-impulsiveness but not inattention (Francx et al., 2015)[83]. The presence of diverse co-morbidity encompasses regional dysregulation of both structural and functional expression that may, or may not, both exacerbate and/or render 'staging-progression' to the disorder.

\section{Conclusions}

Finally, Beare et al. (2016)[84] have observed that ADHD was linked to local proximal regions seen to be more modular and interconnected than in the case of the control groups wherein a decrease in the global, long-range connections, was observed depicting a reduced interchange between local, specialised networks in the ADHD condition. The disorder presents a sub-network of more substantial connectivity embracing bilateral frontostriatal connections in addition to the left occipital, temporal, and parietal regions in which the white matter microstructure was related to with ADHD symptom severity. Under normal conditions, successful neurodevelopmental outcomes emerge from the synchronized and determinative organization of converging neural elements into complex structural and functional networks, the connectome or "wiring-diagram", is a comprehensive map of neural connections in the human brain. Perinatal stress-related/inflammatory symptoms are common in women during pregnancy and are risk factors for neurobehavioral disorders and associated co-morbidities, including ADHD (Flinkkilä et al., 2016; Scheinost et al., 2016)[85,86]. The final outcome of ADHD diagnosis is accompanied by a complex, and discouraging heterogeneous, expression of symptom profiles emerging from altered functional connectivities, default mode network, biomarker identities, absence of necessary dietary ingredients, neuroimmune biomarkers, epigenetic pressures, reward deficiency syndrome and comorbid complications that underlie serious reductions in the quality-of-life and aspirations of the ADHD child and its siblings. As adolescent and adult, the childhood complexities and disruptions may persist to hinder the health trajectory.

\section{References}

1. Archer T, Oscar-Berman M, Blum K (2011) Epigenetics in Developmental Disorder: ADHD and Endophenotypes. J Genet Syndr Gene Ther 2: 104.

2. Archer T, Garcia D (2016) Attention-Deficit/Hyperactivity Disorder: Focus upon Aberrant N-Methyl-D-Aspartate Receptors Systems. Curr Top Behav Neurosci 29: 295-311. 
3. Archer T, Kostrzewa RM (2012) Physical exercise alleviates ADHD symptoms: regional deficits and developmental trajectory. Neurotox Res 21: 195-209.

4. Kessler D, Angstadt M, Sripada C (2016) Growth Charting of Brain Connectivity Networks and the Identification of Attention Impairment in Youth. JAMA Psychiatry. 73: 481-9.

5. Metin B, Krebs RM, Wiersema JR, Verguts T, Gasthuys R, et al. (2015) Dysfunctional modulation of default mode network activity in attention-deficit/hyperactivity disorder. J Abnorm Psychol 124: 208-14.

6. Kessler D, Angstadt M, Welsh RC, Sripada C (2014) Modality-spanning deficits in attention-deficit/hyperactivity disorder in functional networks, gray matter, and white matter. J Neurosci 34: 16555-66.

7a. Sripada CS, Kessler D, Angstadt M (2014) Lag in maturation of the brain's intrinsic functional architecture in attention-deficit/hyperactivity disorder. Proc Natl Acad Sci U S A. 111: 14259-64.

7b. Sripada C, Kessler D, Fang Y, Welsh RC, Prem Kumar K, et al. (2014) Disrupted network architecture of the resting brain in attention-deficit/hyperactivity disorder. Hum Brain Mapp. 35(9):4693-705.

8. Hong SB, Harrison BJ, Fornito A, Sohn CH, Song IC, et al. (2015) Functional dysconnectivity of corticostriatal circuitry and differential response to methylphenidate in youth with attention-deficit/hyperactivity disorder. J Psychiatry Neurosci 40: 46-57.

9. Vilor-Tejedor N, Alemany S, Forns J, Cáceres A, Murcia M, et al (2016) Assessment of Susceptibility Risk Factors for ADHD in Imaging Genetic Studies. J Atten Disord. pii: 1087054716664408.

10. Van Hulst BM, de Zeeuw P, Rijks Y, Neggers SF, Durston S (2016) What to expect and when to expect it: an fMRI study of expectancy in children with ADHD symptoms. Eur Child Adolesc Psychiatry. 10.1007/s00787-016-0921-7

11. Park BY, Hong J, Lee SH, Park H (2016) Functional Connectivity of Child and Adolescent Attention Deficit Hyperactivity Disorder Patients: Correlation with IQ. Front Hum Neurosci. 10: 565.

12. Kang KD, Han DH, Kim SM, Bae S, Renshaw PF (2016) The Correlation Between Cognitive and Movement Shifting and Brain Activity in Children With ADHD. J Atten Disord : 10.1177/1087054716677816.

13. Oldehinkel M, Beckmann CF, Pruim RH, van Oort ES, Franke B, et al. (2016) Attention-Deficit/Hyperactivity Disorder symptoms coincide with altered striatal connectivity. Biol Psychiatry Cogn Neurosci Neuroimaging. 1: 353-63.

14. Bellana B, Liu ZX, Diamond NB, Grady CL, Moscovitch M (2016) Similarities and differences in the default mode network across rest, retrieval, and future imagining. Hum Brain Mapp. 10.1002/hbm.23445.

15. Uytun MC, Karakaya E, Oztop DB, Gengec S, Gumus K, et al. (2016) Default mode network activity and neuropsychological profile in male children and adolescents with attention deficit hyperactivity disorder and conduct disorder. Brain Imaging Behav. 10.1007/s11682-016-9614-6.

16. Broulidakis MJ, Fairchild G, Sully K, Blumensath T, Darekar A, et al. (2016) Reduced Default Mode Connectivity in Adolescents With Conduct Disorder. J Am Acad Child Adolesc Psychiatry. 55: 800-8.

17. Sidlauskaite J, Sonuga-Barke E, Roeyers H, Wiersema JR (2016) Default mode network abnormalities during state switching in attention deficit hyperactivitydisorder. Psychol Med 46: 519-28.

18. Mostert JC, Shumskaya E, Mennes M, Onnink AM, Hoogman M, et al. (2016) Characterising resting-state functional connectivity in a large sample of adults with ADHD. Prog Neuropsychopharmacol Biol Psychiatry 67: 82-91.

19. Zhang J, Cheng W, Liu Z, Zhang K, Lei X, et al. (2016) Neural, electrophysiological and anatomical basis of brain-network variability and its characteristic changes in mental disorders. Brain 139: 2307-21.

20. Lin HY, Tseng WY, Lai MC, Matsuo K, Gau SS (2015) Altered resting-state frontoparietal control network in children with attention-deficit/hyperactivity disorder. J Int Neuropsychol Soc 21: 271-84.

21. Wang XH, Li L (2015) Altered temporal features of intrinsic connectivity networks in boys with combined type of attention deficit hyperactivity disorder. Eur J Radiol 84: 947-54.

22. Van Rooij D, Hartman CA, Mennes M, Oosterlaan J, Franke B, et al. (2015) Altered neural connectivity during response inhibition in adolescents with attention-deficit/hyperactivity disorder and their unaffected siblings. Neuroimage Clin 7: 325-35.

23. Lang EW, Tomé AM, Keck IR, Górriz-Sáez JM, Puntonet CG (2012) Brain connectivity analysis: a short survey. Comput Intell Neurosci $2012: 412512$.

24. Yo TS, Anwander A, Descoteaux M, Fillard P, Poupon C, et al. (2009) Quantifying brain connectivity: a comparative tractography study. Med Image Comput Comput Assist Interv 12: 886-93.

25. Žarić G, Correia JM, Fraga González G, Tijms J, van der Molen MW, et al. (2016) Altered patterns of directed connectivity within the reading network of dyslexic children and their relation to reading dysfluency. Dev Cogn Neurosci 23: 1-13.

26. Liuzzi L, Gascoyne LE, Tewarie PK, Barratt EL, Boto E, et al. (2016) Optimising experimental design for MEG resting state functional connectivity measurement. Neuroimage :10.1016/j.neuroimage.2016.11.064.

27. Trotta N, Archambaud F, Goldman S, Baete K, Van Laere K, et al. (2016) Functional integration changes in regional brain glucose metabolism from childhood to adulthood. Hum Brain Mapp 37: 3017-30.

28. Jann K, Hernandez LM, Beck-Pancer D, McCarron R, Smith RX, et al. (2015) Altered resting perfusion and functional connectivity of default mode network in youth with autism spectrum disorder. Brain Behav : 10.1002/brb3.358.

29. Hung NC, Lin SH, Chi MH, Lin CH, Chen PS, et al. (2016) Poor performance of fine motor activity among biological parents of children with attentiondeficit/ hyperactivity disorder. Kaohsiung J Med Sci 32: 630-3.

30. Schrantee A, Mutsaerts H, Bouziane C, Tamminga H, Bottelier MA, et al. (2016) The age-dependent effects of a single-dose methylphenidate challenge on cerebral perfusion in patients with attention-deficit/hyperactivity disorder. Neuroimage Clin 13: 123-9.

31. Ziegler S, Pedersen ML, Mowinckel AM, Biele G (2016) Modelling ADHD: A review of ADHD theories through their predictions for computational models of decision-making and reinforcement learning. Neurosci Biobehav Rev. 71: 633-56.

32. Miller EM, Pomerleau F, Huettl P, Gerhardt GA, Glaser PE (2014) Aberrant glutamate signaling in the prefrontal cortex and striatum of the spontaneously hypertensive rat model of attention-deficit/hyperactivity disorder. Psychopharmacology (Berl) 231: 3019-29.

33. Fredriksson A, Archer T (2002) Functional alteration by NMDA antagonist: effects of L-Dopa, neuroleptic drugs and postnatal administration. Amino Acids 23: 111-32.

34. Fredriksson A, Archer T (2003) Hyperactivity following postnatal NMDA antagonist treatment: reversal by D-amphetamine. Neurotox Res 5: 549-64. 
35. Fredriksson A, Archer T (2004) Neurobehavioral deficits associated with apoptotic neurodegeneration and vulnerability for ADHD. Neurotox Res 6: 435-56. 36. Fredriksson A, Archer T, Alm H, Gordh T, Eriksson P (2004) Neurofunctional deficits and prolonged apoptosis by neonatal NMDAantagonist administration. Behav Brain Res 153: 367-76.

37. Bonhomme V, Vanhaudenhuyse A, Demertzi A, Bruno MA, Jaquet O, et al. (2016) Resting-state Network-specific Breakdown of Functional Connectivity during Ketamine Alteration of Consciousness in Volunteers. Anesthesiology 125: 873-88.

38. Hove MJ, Zeffiro TA, Biederman J, Li Z, Schmahmann J, et al. (2015) Postural sway and regional cerebellar volume in adults with attention-deficit/hyperactivitydisorder. Neuroimage Clin 8: 422-8.

39. Kucyi A, Hove MJ, Biederman J, Van Dijk KR, Valera EM (2015) Disrupted functional connectivity of cerebellar default network areas in attention-deficit/ hyperactivity disorder. Hum Brain Mapp 36: 3373-86.

40. Cai W, Chen T, Szegletes L, Supekar K, Menon V (2015) Aberrant Cross-Brain Network Interaction in Children With Attention-Deficit/HyperactivityDisorder and Its Relation to Attention Deficits: A Multisite and Cross-Site Replication Study. Biol Psychiatry : 10.1016/j.biopsych.2015.10.017.

41. Biskup CS, Helmbold K, Baurmann D, Klasen M, Gaber TJ, et al. (2016) Resting state default mode network connectivity in children and adolescents with ADHD after acute tryptophan depletion. Acta Psychiatr Scand 134: 161-71.

42. Vanicek T, Kutzelnigg A, Philippe C, Sigurdardottir HL, James GM, et al (2016) Altered interregional molecular associations of the serotonin transporter in attention deficit/hyperactivity disorder assessed with PET. Hum Brain Mapp : 10.1002/hbm.23418.

43. Battel L, Kieling RR, Kieling C, Anés M, Aurich NK, et al. (2016) Intrinsic Brain Connectivity Following Long-Term Treatment with Methylphenidate in Children with Attention-Deficit/Hyperactivity Disorder. J Child Adolesc Psychopharmacol. 26: 555-61.

44. Modestino EJ, Blum K, Oscar-Berman M, Gold MS, Duane DD, et al. (2015) Reward Deficiency Syndrome: Attentional/Arousal Subtypes, Limitations of Current Diagnostic Nosology, and Future Research. J Reward Defic Syndr 1: 6-9.

45. Blum K, Sheridan PJ, Wood RC, Braverman ER, Chen TJ, et al. (1996) The D2 dopamine receptor gene as a determinant of reward deficiency syndrome. J R Soc Med 89: 396-400.

46. Blum K, Braverman ER, Holder JM, Lubar JF, Monastra VJ, et al. (2000) Reward deficiency syndrome: a biogenetic model for the diagnosis and treatment of impulsive, addictive, and compulsive behaviors. J Psychoactive Drugs 32: 1-112.

47. Blum K, Chen AL, Braverman ER, Comings DE, Chen TJ, et al. (2008a) Attention-deficit-hyperactivity disorder and reward deficiency syndrome. Neuropsychiatr Dis Treat 4: 893-918.

48. Blum K, Chen AL, Chen TJ, Braverman ER, Reinking J, et al. (2008b) Activation instead of blocking mesolimbic dopaminergic reward circuitry is a preferred modality in the long term treatment of reward deficiency syndrome (RDS): a commentary. Theor Biol Med Model : 10.1186/1742-4682-5-24.

49. Blum K, Chen TJ, Chen AL, Madigan M, Downs BW, et al. (2010) Do dopaminergic gene polymorphisms affect mesolimbic reward activation of music listening response? Therapeutic impact on Reward Deficiency Syndrome (RDS). Med Hypotheses 74: 513-20.

50. Leventakou V, Micali N, Georgiou V, Sarri K, Koutra K, et al. (2016) Is there an association between eating behaviour and attention-deficit/hyperactivity disorder symptoms in preschool children? J Child Psychol Psychiatry 57: 676-84.

51. Brewerton TD, Duncan AE (2016) Associations between Attention Deficit Hyperactivity Disorder and Eating Disorders by Gender: Results from the National Comorbidity Survey Replication. Eur Eat Disord Rev 24: 536-40.

52. Wunderli MD, Vonmoos M, Niedecker SM, Hulka LM, Preller KH, et al. (2016) Cognitive and emotional impairments in adults with attention-deficit/hyperactivity disorder and cocaine use. Drug Alcohol Depend 163: 92-9.

53. Miguel CS, Martins PA, Moleda N, Klein M, Chaim-Avancini T, et al. (2016) Cognition and impulsivity in adults with attention deficit hyperactivity disorder with and without cocaine and/or crack dependence. Drug Alcohol Depend 160: 97-104.

54. Capusan AJ, Bendtsen P, Marteinsdottir I, Larsson H (2016) Comorbidity of Adult ADHD and Its Subtypes With Substance Use Disorder in a Large Population-Based Epidemiological Study. J Atten Disord : 10.1177/1087054715626511

55. Caye A, Swanson J, Thapar A, Sibley M, Arseneault L, et al. (2016) Life Span Studies of ADHD-Conceptual Challenges and Predictors of Persistence and Outcome. Curr Psychiatry Rep 18: 111.

56. Kim J, Park H, Yu SL, Jee S, Cheon KA, et al. (2016) Effects of high-frequency repetitive transcranial magnetic stimulation (rTMS) on spontaneously hypertensive rats, an animal model of attention-deficit/hyperactivity disorder. Int J Dev Neurosci 53: 83-9.

57. Ruocco LA, Treno C, Gironi Carnevale UA, Arra C, Boatto G, et al. (2015) Immunization with DISC1 protein in an animal model of ADHD influences behavior and excitatory amino acids in prefrontal cortex and striatum. Amino Acids 47: 637-50.

58. Baca M, Allan AM, Partridge LD, Wilson MC (2013) Gene-environment interactions affect long-term depression (LTD) through changes in dopamine receptor affinity in Snap25 deficient mice. Brain Res 1532: 85-98.

59. Rivero O, Selten MM, Sich S, Popp S, Bacmeister L, et al. (2015) Cadherin-13, a risk gene for ADHD and comorbid disorders, impacts GABAergic function in hippocampus and cognition. Transl Psychiatry : 10.1038/tp.2015.152.

60. Wu LH, Cheng W, Yu M, He BM, Sun H, (2016) Nr3C1-Bhlhb2 Axis Dysregulation Is Involved in the Development of Attention DeficitHyperactivity. Mol Neurobiol : 10.1007/s12035-015-9679-z.

61. Xu Y, Chen XT, Luo M, Tang Y, Zhang G, et al. (2015) Multiple epigenetic factors predict the attention deficit/hyperactivity disorder among the Chinese Han children. J Psychiatr Res 64: 40-50.

62. Lien GW, Huang CC, Shiu JS, Chen MH, Hsieh WS, et al. (2016) Perfluoroalkyl substances in cord blood and attention deficit/hyperactivity disorder symptoms in seven-year-old children. Chemosphere 156:118-27.

63. Mostafalou S, Abdollahi M (2016) Pesticides: an update of human exposure and toxicity. Arch Toxicol : 10.1007/s00204-016-1849-x.

64. Motaghinejad M, Motevalian M, Shabab B (2016) Effects of chronic treatment with methylphenidate on oxidative stress and inflammation in hippocampus of adult rats. Neurosci Lett 619: 106-13.

65. Shah K, Lahiri DK (2016) A Tale of the Good and Bad: Remodeling of the Microtubule Network in the Brain by Cdk5. Mol Neurobiol : 10.1007/s12035-0169792-7.

66. Oliveira-Pinto J, Paes-Branco D, Cristina-Rodrigues F, Krahe TE, Manhães AC, et al. (2015) GABAA overactivation potentiates the effects of NMDA blockade during the brain growth spurt in eliciting locomotor hyperactivity in juvenile mice. Neurotoxicol Teratol 50: 43-52. 
67. Di Miceli M, Gronier B (2016) Psychostimulants and atomoxetine alter the electrophysiological activity of prefrontal cortex neurons, interaction with catecholamine and glutamate NMDA receptors. Psychopharmacology 232: 2191-205.

68. Miller EM, Quintero JE, Pomerleau F, Huettl P, Gerhardt GA, et al. (2015) Simultaneous glutamate recordings in the frontal cortex network with multisite biomorphic microelectrodes: New tools for ADHD research. J Neurosci Methods 252:75-9.

69. Fluegge $\mathrm{K}$ (2016) Does environmental exposure to the greenhouse gas, $\mathrm{N}<\mathrm{sub}>2</$ sub $>\mathrm{O}$, contribute to etiological factors in neurodevelopmental disorders? A mini-review of the evidence. Environ Toxicol Pharmacol 47: 6-18.

70. Brikell I, Kuja-Halkola R, Larsson JO, Lahey BB, Kuntsi J, et al. (2016) Relative Immaturity in Childhood and Attention-Deficit/Hyperactivity Disorder Symptoms From Childhood to Early Adulthood: Exploring Genetic and Environmental Overlap Across Development. J Am Acad Child Adolesc Psychiatry 55: 886-95.

71. Homberg JR, Kyzar EJ, Scattoni ML, Norton WH, Pittman J, et al. (2016) Genetic and environmental modulation of neurodevelopmental disorders: Translational insights from labs to beds. Brain Res Bull 125: 79-91.

72. Ferrer M, Andión Ó, Calvo N, Ramos-Quiroga JA, Prat M, et al. (2016) Differences in the association between childhood trauma history and borderline personality disorder or attention deficit/hyperactivity disorder diagnoses in adulthood. Eur Arch Psychiatry Clin Neurosci : 10.1007/s00406-016-0733-2.

73. Chen Q, Brikell I, Lichtenstein P, Serlachius E, Kuja-Halkola R, et al. (2016) Familial aggregation of attention-deficit/hyperactivity disorder. J Child Psychol Psychiatry : 10.1111/jcpp.12616.

74. Yin P, Hou X, Qin Q, Deng W, Hu H, et al. (2016) Genetic and Environmental Influences on the Mental Health of Children: A Twin Study. J Psychosoc Nurs Ment Health Serv 54: 29-34.

75. Aronson B (2016) Peer influence as a potential magnifier of ADHD diagnosis. Soc Sci Med 168: 111-19.

76. Joo H, Lim MH, Ha M, Kwon HJ, Yoo SJ, et al. (2016) Secondhand Smoke Exposure and Low Blood Lead Levels in Association With Attention-DeficitHyperactivity Disorder and Its Symptom Domain in Children: A Community-Based Case-Control Study. Nicotine Tob Res 19: 94-101.

77. Lopez-Vicente M, Forns J, Suades-Gonzalez E, Esnaola M, Garcia-Esteban R, et al. (2016) Developmental trajectories in primary schoolchildren using n-back task. Front Psychol 7: 716.

78. Smith E, Meyer BJ, Koerting J, Laver-Bradbury C, Lee L, et al. (2016) Preschool hyperactivity specifically elevates long-term mental health risks more strongly in males than females: a prospective longitudinal study through to young adulthood. Eur Child Adolesc Psychiatry : 10.1007/s00787-016-0876-8.

79. Vilor-Tejedor N, Cáceres A, Pujol J, Sunyer J, González JR (2016) Imaging genetics in attention-deficit/hyperactivity disorder and related neurodevelopmental domains: state of the art. Brain Imaging Behav : 10.1007/s11682-016-9663-x

80. Grant R, Guest J (2016) Role of Omega-3 PUFAs in Neurobiological Health. Adv Neurobiol 12: 247-74.

81. Cacabelos R, Torrellas C, Fernández-Novoa L, López-Muñoz F (2016) Histamine and Immune Biomarkers in CNS Disorders. Mediators Inflamm : $10.1155 / 2016 / 1924603$

82. von Rhein D, Oldehinkel M, Beckmann CF, Oosterlaan J, Heslenfeld D, et al. (2016) Aberrant local striatal functional connectivity in attention-deficit/hyperactivity disorder. J Child Psychol Psychiatry 57: 697-705.

83. Francx W, Oldehinkel M, Oosterlaan J, Heslenfeld D, Hartman CA, et al. (2015) The executive control network and symptomatic improvement in attention-deficit/hyperactivity disorder. Cortex 73: 62-72.

84. Beare R, Adamson C, Bellgrove MA, Vilgis V, Vance A, et al. (2016) Altered structural connectivity in ADHD: a network based analysis. Brain Imaging Behav : 10.1007/s11682-016-9559-9.

85. Flinkkilä E, Keski-Rahkonen A, Marttunen M, Raevuori A (2016) Prenatal Inflammation, Infections and Mental Disorders. Psychopathology 49: $317-33$.

86. Scheinost D, Sinha R, Cross SN, Kwon SH, Sze G, et al. (2016) Does prenatal stress alter the developing connectome? Pediatr Res : $10.1038 /$ pr.2016.197. 